\title{
On the use of the reconstructive technique: criticisms, comments and questions
}

\author{
S. L. Jensen*, B. D. Robbins, S. S. Bell \\ Department of Biology, University of South Florida, Tampa, Florida 33620-5150, USA
}

Our original study conducted on seagrass populations in Rabbit Key Basin (RKB) (Jensen et al. 1996) had 2 main purposes: (1) to compare the results of a seagrass demographic model when samples were taken in multiple locations as well as when data collection procedures were varied, and (2) to test whether predictions made about seagrass population changes by Durako (1994) were adequately verified by subsequent sampling. These objectives were independent of one another but, combined, contributed to an assessment of the application of the reconstructive technique described by Duarte et al. (1994) and utilized by Durako (1994).

Durako \& Duarte (1997) (hereafter referred to as $D \& D$ ) have criticised our paper (Jensen et al. 1996). Many of their comments are addressed below. We first revisit issues of methodology and sampling to show that our procedures were appropriate and our data interpretation fair. We then evaluate claims made by D\&D of misjudgement, examine purported biases and consider the possible impact of biases on both results and conclusions. Finally, we address a possible fundamental difference in perception as to the critical questions inherent in effective management of coastal resources, including questions that may be necessary for evaluation of the reconstructive technique as a coastal management tool.

$D \& D$ state that '... specific comparison of shoot densities measured by Durako (1994) with those reported by Jensen et al. (1996) represents an inappropriate test of the earlier study's predictions...' because we were '...using data collected over 4 yr later, from different sites, during a different time of year, and using different sample collecting and handling techniques'. Each of these charges will be addressed.

By extending the 'prediction' to greater than $1 \mathrm{yr}$, D\&D state that we violate the intent of Durako (1994).

•E-mail: jensen@chuma.cas.ust.edu
In Jensen et al. (1996), we deliberately avoided criticism of the 1 yr forecast of shoot densities made by Durako (1994), resulting from the reconstructive model. Instead we focused upon testing the conclusion of the study, which stated: 'Predictions based on estimates of shoot recruitment and mortality suggest that net loss of short-shoots will continue and that even greater deterioration of Thalassia testudinum populations in Florida Bay may be expected in the near future' (Durako 1994, p. 65). There is no question that such a statement extends the work beyond the sample sites in which Durako (1994) worked previously, and that the phrase 'expected in the near future' (albeit very imprecise) reaffirms that the prediction was not limited to a single year forecast as argued by $D \& D$.

Testing the prediction of continued population decline necessitated a variety of judgement calls because the information on rates of shoot density decline in Durako (1994) was not obvious. We established a generous window of expected population decline, using the following statements: 'Shoot densities in 1991 are predicted to be 15 to $49 \%$ lower than those in 1990, with the greatest declines occurring in the JKB [Johnson Key Basin] and RKB populations' (Durako 1994, p. 64), 'Although mortality rates have increased in all 3 basins, the decline in shoot densities should be faster in JKB and RKB, where population half-lives showed the highest drop' (Durako 1994, p. 65) and 'The demographic analyses performed here revealed that the RNK [Rankin Lakel population is already severely affected by die-off, and it predicts that declines in the other 2 populations [JKB and RKB] are soon to follow' (Durako 1994, p. 65). Thus we surmised that 15 and $49 \%$ should bracket the rate of seagrass decline in RKB. Although D\&D fault our logic, stating that our study was guilty of '.misinterpreting the prediction values which were formulated for 3 different basins, not as ranges for Rabbit Key Basin', they avoid noting that our selection of 15 to $49 \%$ annual 
rate of decline successfully included the predicted value for RKB. They also fail to recognize that the bounds of our arbitrarily selected window of decline had little impact on our conclusions given that the population densities of seagrass at our sample sites within RKB were simply not lower in 1994 than those in 1990 (see Jensen et al. 1996, Fig. 2).

Claims that Durako's (1994) use of a stratified sampling renders our comparison with his data invalid should be dismissed outright. Durako's (1994) stratified sampling is only illusory because samples from all 'strata' were lumped for analyses. Our study did not specifically target nor exclude sites that were exclusively die-off areas in order to obtain the best possible estimate of population density within our sampled area of RKB. We question the appropriateness of a sampling design targeting only areas with die-off for assessing the general health of Florida Bay seagrass, be they in the present or near future. We do not imply that there are not areas within RKB experiencing die-off; we do show that an unbiased sampling did not provide results that confirmed the conclusion of Durako (1994).

D\&D, citing work by Durako (1995), claim that our seagrass samples from July cannot be compared with samples collected in April because of expected seasonal differences. We examined the impact of possible bias on shoot density estimates using the information presented in Durako (1995; Fig. 1) which indicates that Thalassia testudinum shoot densities decline seasonally in RKB from April through October. Our July values are thus biased toward lower densities than that which would be expected in April and so reaffirm our conclusion that the densities of seagrass in Rabbit Key Basin are not declining as predicted in Durako (1994).

D\&D claim that our sampling procedure and handling of samples do not allow for comparison between studies. This is a curious statement. Our 1994 calculation of total shoot densities per $\mathrm{m}^{2}$ from standard-size cores should be directly comparable with 1990 estimates of density per $\mathrm{m}^{2}$ based upon calculations from smaller quadrats unless data presented in Durako (1994) do not include all shoots in his sample enumeration. Therefore the above challenges to our demographic comparisons do not necessitate a modification of our original discussion.

In preparing our original data for analyses, we sought counsel from both co-authors of $D \& D$ with respect to employment of the reconstructive technique. Many of their comments were helpful in performing the analyses but a few procedural questions remained unanswered or were further confused by their input. For example, approaches for estimating mortality were inconsistent with respect to the portion of the age distribution to be included in the curve-fitting calculation used to estimate mortality. One author stated that all the information in the age distribution should be used, i.e. every age class contributes to the final estimate of mortality (C. M. Duarte pers. comm.) while the other author used only subjectively selected cohort peaks in determination of the mortality estimate of the entire population (M. J. Durako pers. comm.). We chose to use the first method, which allows for a more conservative estimate of mortality.

$D \& D$ take issue with our use of a plastochron interval (PI) published in Durako (1994; Table 1), rather than calculating our own, on the basis that '...durations of PIs can exhibit considerable spatial and temporal variability resulting from environmental influences...' We are grateful for reiteration of this point, as this was an underlying theme of our paper (Jensen et al. 1996). According to Duarte et al. (1994, p. 198), 'Interannual variability in $\mathrm{PI}$ is usually much smaller than seasonal variability, such that the linearity between PI and absolute time assumed by these age determinations is best supported at interannual time scales...' Accordingly, the 1990 PI should be appropriate for our use in 1994, since we were testing the model on a yearly basis, rather than looking for seasonal differences. The implications of possible variation in the PI are addressed neither by Durako (1994), Duarte et al. (1994) nor D\&D, and this is an area in need of mathematical exploration. For example, variation in the PI will alter the chronological age assigned to shoots in the sampled population, shifting the number of shoots considered to be recruits and ultimately altering demographic characteristics, such as gross and net recruitment, assigned to a population (see Jensen et al. 1996, Fig. 4). Mortality rates are calculated by the slope of the age distribution (cf. Duarte et al. 1994), which is not affected by PI value. Jensen et al. (1996) looked for effects of variability in demographic characteristics, and by holding PI constant avoided specifically testing the effects of seasonal and spatial changes in PI. Thus we feel that the use of a single, published value of the $\mathrm{PI}$ is justified in the context of our study.

In order to compare demographic characteristics among sites, our study was conducted in 3 different sample sites encompassing a 52.5 ha area within the $>3500$ ha RKB. D\&D state that 'The gross recruitment and mortality rates in Table 2 of Jensen et al. (1996) are not significantly different among sites...' and that '... [our] data provide little evidence that spatial variability is an important factor potentially biasing the demographic predictions'. The basis of their statistical evaluation was not revealed but further reading of our paper (Jensen et al. 1996, p. 269-271) will show that this issue has been addressed. These sampling sites did not cross biotic or environmental gradients (see Zieman et al. 1989), as was addressed in Jensen et al. (1996, p. 269), contrary to suggestions by D\&D. 
Change in population size or density over time is the most straightforward method of measuring growth or decline of a population. In addition to density, Jensen et al. (1996) compared demographic data (mean and maximum age, recruitment and mortality rates) collected in 1994 to those collected in 1989 and 1990, as presented in Durako (1994). D\&D state that 'because the distributions of shoot age are strongly skewed (see Fig. 3 in Jensen et al. 1996), mean values are an inappropriate measure of central tendency.. ', and that 'A more appropriate comparison of the central tendency in shoot age distributions with differing sample size should be based on the median...' We agree: the calculation of mean shoot age was employed to enable explicit comparisons with samples from RKB in Durako (1994), in which mean age was used to characterize a set of highly skewed data (see Durako 1994, Fig. 5).

A significant indictment leveled by $D \& D$ is that '...results obtained by Jensen et al. (1996) are remarkably similar to those of Durako (1994) and an objective evaluation of the data should not have failed to note this similarity'. Again, a careful reading of Jensen et al. (1996) reveals that we compared our 5 demographic parameters (maximum age, gross recruitment, mortality, net recruitment and population 'half-life') for 1994 data (Jensen et al. 1996; Table 2), with complementary values presented by Durako for both 1989 and 1990 (a total of 10 comparisons). Three of 10 values matched those in Durako (1994) and were acknowledged (Jensen et al. 1996, p. 271). Seven others differed by 28 to $200 \%$. We thus concluded that our results differed from those of Durako (1994), while D\&D contend that this is not so. We cannot resolve this problem of perspective but the accusation of lack of objectivity is misplaced.

D\&D impugn our statistical comparison of age distributions: 'The attached-shoot population is a subsample of the total-shoot population and therefore is not an independent sample ... nonindependence of samples violates the most fundamental of statistical assumptions and invalidates any evaluation of statistical significance for attached vs total shoots (Tables $1 \& 3$ in Jensen et al. (1996)'. One of the stated objectives of our paper was to test whether the exclusion of unattached shoots from data analyses had an impact on the evaluation of demographic information. Analysis of the effect of data censoring by exclusion of unattached shoots required that values from a subsample be compared to the larger sample. By narrowing the inference space of the questions asked, lack of independence can be addressed. Specifically, we asked whether the age distribution of attached shoots in our sample mirrored that of the total shoots (which included both attached and unattached shoots; Jensen et al. 1996). Revisiting the same components as in Tables 1 \& 3 of our paper (Jensen et al. 1996), data from each sampling station were analyzed using a Monte Carlo without replacement procedure (Fishman 1996), at a critical value of $p<0.05$. Our results are in accordance with findings in Jensen et al. (1996), i.e. age distributions between the sub-samples and their respective total samples were not significantly different (see Table 3 , Jensen et al. 1996). Mean age of shoots was significantly different between subsample and sample, as found previously (see Table 1, Jensen et al. 1996). When analyses were expanded to include median age (cf. Duarte et al. 1994), improving upon the data analyses in both Durako (1994) and Jensen et al. (1996), median age was also found to be significantly different in all cases (see Jensen et al. 1996, Table 1, for tests performed). Using Monte Carlo techniques, all shoot density comparisons were also significantly different $(p<0.05)$, again supporting prior results (Jensen et al. 1996, Table 1). The criticism regarding choice of $p$ value when performing multiple comparisons raised by $D \& D$ is unwarranted. Although $\alpha$ levels can be adjusted using a Bonferroni correction when making multiple comparisons, the level at which a Type I error rate is controlled is arbitrary and left to the choice of the experimenter (Bogartz 1994). Our conclusions that significant spatial heterogeneity exists in demographic characteristics among sites remains well supported by statistical analysis, as does our contention that subsamples may not provide an adequate representation of some demographic features of the total sample.

We agree with $D \& D$ that using age-structured matrix models may not be most appropriate for seagrasses; stage-structured models are what have historically been tested and found to be appropriate for clonal plants (cf. Sarukhán \& Harper 1973), including seagrasses (Ewanchuk 1995). Stage-structured matrix. models have been used extensively on population dynamics of terrestrial clonal plants such as grasses and long-lived species (e.g. Nault \& Gagnon 1993); the matrix models also incorporate multiple modes of reproduction (e.g. Sarukhán \& Gadgil 1974) in the estimates of population dynamics. It is unclear to us why this has been deemed 'too demanding' (D\&D) for seagrass biologists.

Divergence in convictions between ourselves and $D \& D$ is implicit in their comments on our paper. D\&D state that '...assessment and prediction, rather than understanding, are more frequently the primary goals for seagrass ecologists', particularly for resource managers. This divergence represents a fundamental rift among many ecologists (e.g. Peters 1991); we do not profess to resolve the rift, but we re-emphasize that understanding factors influencing population dynamics will be necessary for effective management. This understanding is particularly indispensable in the case 
of seagrass die-off, where there are many stages of the life cycle during which die-off may be affecting the populations. Without knowledge of the confounding factors of multiple modes of reproduction, and seasonal and spatial variability in demographic characteristics, any attempts to pinpoint a cause of, and later, control, die-off may be futile. To this end, appropriate demographic analyses may include adaptations of multiple-decrement life tables or key factor analysis.

$D \& D$ argue that the use of reconstructive techniques currently offers the only practical alternative to assessing seagrass demography of long-lived species...' Jensen et al. (1996) acknowledge that collection of demographic information can provide some detailed insight into the nature of response of populations under a variety of conditions, and that collection of such data certainly improves our understanding of seagrass ecology. It remains that the reconstructive technique is simply that: reconstruction of the history of an individual plant. Proper sampling may allow reconstruction of a population's history and give clues to changing environmental conditions and the population's response to those changes. If historical population dynamics are known, then extrapolations of population dynamics into the future may be made, assuming constant or predictable environmental conditions. In the case of as-yet unpredictable seagrass die-off, however, environmental conditions must be changing by virtue of the fact that die-off is occurring; hence the ability to forecast populations is severely restricted. Recovery rates of die-off patches may be projected using data from the reconstructive technique (Duarte et al. 1994), but forecasting population dynamics using the one-time sampling inherent in the reconstructive technique (cf. Durako 1994) may have severe limitations as discussed in Jensen et al. (1996). This is not a problem particular to seagrass; limitations on snapshot-sampling for vital statistics of a population have been widely acknowledged in ecology (see Krebs 1994 for a textbook example).

We argue that embracing the reconstructive technique as the provider of the knowledge necessary to assess and manage these important coastal ecosystems' (D\&D) is premature. This is an issue that will mandate careful consideration of the methodology employed (such as the initial attempt by Jensen et al. 1996), as well as its appropriate application. Clearly, asking pertinent questions about accuracy and limitations of a technique must be a fundamental concern to resource managers as potential users. Ease of use is certainly an attractive feature of a resource assessment technique as argued by $D \& D$; the attractiveness is diminished if flaws in the technique produce data that are easily collected but of questionable value. Rigorous testing of any new technique may be done by resource managers themselves or even by academic researchers that, as D\&D declare, 'require extensive local resources'

According to Duarte et al. (1994), one of the practical aspects of the reconstructive technique is that demographic information can be gathered from a single or few sampling events. We submit that it remains to be determined whether reconstructive techniques improve upon other methods used to evaluate seagrass health such as more frequent, periodic monitoring programs, including remote sensing (Robbins in press). In closing, we offer 2 pertinent questions that address the issue of practicality and invite debate: Is a predictive population model that requires field sampling and extensive laboratory processing particularly useful if it is restricted in use to a $1 \mathrm{yr}$ forecast, especially when the model is touted as a labor-saving device? If a model must be reverified and its anomalies rejustified year after year, would investigators be better served to continue periodic monitoring of the population, which provides the most accurate and detailed information, perhaps for the same (or even less) amount of effort? Discussions on these issues will only be progressive if facts supported by data, and not opinion, are the basis of discourse.

Acknowledgements. We thank the editor for the opportunity to prepare this response. Discussions with S. Service and E. D. McCoy provided perspective and technical help in the preparation of this reply. This study was supported in part by NOAA Coastal Ocean Program-Estuarine Habitat Research Program, and an NSF DEB grant 9318130, S. S. Bell, principal investigator.

\section{LJTERATURE CITED}

Bogartz RS (1994) An introduction to the analysis of variance. Praeger Publishers, Westport, CT

Duarte CM, Marba N, Agawin N, Cebrian J, Enriquez S, Fortes MD, Gallegos ME, Merino M, Olesen B. SandJensen K, Uri J, Vermaat J (1994) Reconstruction of seagrass dynamics: age determinations and associated tools for the seagrass ecologist. Mar Ecol Prog Ser 107 195-209

Durako MJ (1994) Seagrass die-off in Florida Bay (USA) changes in shoot demographic characteristics and population dynamics in Thalassia testudinum. Mar Ecol Prog Ser 110:59-66

Durako MJ (1995) Indicators of seagrass ecological condition an assessment based on spatial and temporal changes. In Dyer KR, Orth RJ (eds) Changes in fluxes in estuaries implications for science to management. Olsen and Olsen, Fredensborg, p 261-266

Durako MJ, Duarte CM (1997) On the use of reconstructive aging techniques for assessing seagrass demography: a critique of the model test of Jensen et al. (1996). Mar Ecol Prog Ser 146:297-303

Ewanchuk P (1995) The relative importance of sexual versus asexual reproduction in eelgrass (Zostera marina L.) population growth. MSc thesis, San Diego State University

Fishman GS (1996) Monte Carlo: concepts, algorithms, and 
applications. Springer Series in Operations Research, New York

Jensen SL, Robbins BD, Bell SS (1996) Predicting population decline: seagrass demographics and the reconstructive technique. Mar Ecol Prog Ser 136:267-276

Krebs CJ (1994) Ecology: the experimental analysis of distribution and abundance, 4th edn. Harper Collins College Publishers, New York

Nault A, Gagnon D (1993) Ramet demography of Allium trscoccum, a spring ephemeral, perennial forest herb. J Ecol 81:101-119

Peters RH (1991) A critique for ecology. Cambridge University Press, Cambridge
Robbins BD (in press) Quantifying temporal change in seagrass using GIS and low resolution aerial photography Aquat Bot

Sarukhán J, Harper JL (1973) Studies on plant demography Ranunculus repens $\mathrm{L}$., $R$. bulbosus $\mathrm{L}$ and $R$. acris $\mathrm{L}$. I. Population flux and survivorship. J Ecol 61:675-716

Sarukhán J. Gadgil M (1974) Studies on plant demography Ranunculus repens L., $R$. bulbosus L. and $R$. acris L. III. A mathematical model incorporating multiple modes of reproduction. J Ecol 62:921-936

Zieman JC, Fourqurean JW, Iverson RL (1989) Distribution, abundance and productivity of seagrasses and macro algae in Florida Bay. Bull Mar Sci 44:292-311 